\title{
Nasosinus Metastases from Breast Cancer: A Review of Two Cases
}

\section{Papa Macoumba Gaye ${ }^{1 *}$, Mouhamadou Bachir Ba1, Fatimatou Néné Sarr¹, Fallou Niang², Maimouna Mané1, Franck Chouamou Aurélien Chemega ${ }^{1}$}

\author{
${ }^{1}$ Radiotherapy Department of Dalal Jamm Hospital, Cheikh Anta Diop University of Dakar, Dakar, Senegal \\ ${ }^{2}$ Oto-Rhino-Laryngology Department of Fann Hospital, Cheikh Anta Diop University of Dakar, Dakar, Senegal \\ Email: *macoumba.gaye@gmail.com
}

How to cite this paper: Gaye, P.M., Ba, M.B., Sarr, F.N., Niang, F., Mané, M. and Chemega, F.C.A. (2021) Nasosinus Metastases from Breast Cancer: A Review of Two Cases. Advances in Breast Cancer Research, 10, 133-140.

https://doi.org/10.4236/abcr.2021.104011

Received: June 29, 2021

Accepted: August 14, 2021

Published: August 17, 2021

Copyright $\odot 2021$ by author(s) and Scientific Research Publishing Inc. This work is licensed under the Creative Commons Attribution International License (CC BY 4.0).

http://creativecommons.org/licenses/by/4.0/

\begin{abstract}
Context: Breast cancer affects more than 2 million women a year worldwide. Improved treatments have made it possible to increase survival rates with more and more patients having time to develop secondary locations. Nasosinus metastases from this cancer are rare. Two recently treated breast cancer patients developed metastases to the sphenoidal and maxillary sinuses. These cases gave rise to an examination of the incidence of this disease, its diagnosis, the therapeutic means envisaged and the fate of these patients. Method: We report the diagnostic and therapeutic data of two patients suffering from breast cancer and presenting metastases at the nasosinus level, with a focus on the interest of radiotherapy in palliative care. Results: The two patients aged 56 and 60 presented in the course of their breast cancer secondary localizations in the sphenoidal and maxillary sinus confirmed by endoscopy and histology. All the cases presented massive lesions associated with other secondary bone locations in particular. After local radiotherapy and systemic chemotherapy treatment, one patient died 5 months after her diagnosis and the other was alive 16 months later. Conclusion: Nasal and sinus metastases from breast cancer are rare and have a poor prognosis as disseminated disease, they do not respond well to conventional systemic therapies even if palliative radiotherapy allows local control.
\end{abstract}

\section{Keywords}

Cancer, Breast, Nasosinus Metastasis, Senegal

\section{Introduction}

Breast cancer is a major public health concern with an increasing incidence in 
recent years [1]. Improved treatments have made it possible to increase survival rates with more and more patients having time to develop secondary locations. Nasosinus metastases from breast cancer are exceptional. Only a few cases of paranasal breast cancer metastases have ever been published [2] [3] [4]. The mechanism of paranasal sinus metastases is unclear. Hematogenous spread through the spine via the venous plexus has been advanced [5]. The Valsalva maneuver can cause retrograde flow through a low-pressure, valveless system, which communicates with the venous system of the thorax and transports tumor emboli to the pterygoid plexus and paranasal sinuses [5] [6]. We report the diagnostic and therapeutic data of two patients suffering from breast cancer and having presented metastases at the nasosinus level in the course of their disease.

\section{Patients and Observation}

\subsection{Clinical Case 1}

A 56-year-old woman was admitted to the Oto-Rhino-Laryngology department of the National University Hospital (NUH) of Fann and then referred to the radiotherapy department of the NUH Dalal Jamm in Dakar. She had presented a symptomatology made up of hemicranias resistant to analgesics and unilateral nasal obstruction of chronic evolution. The clinical examination had revealed exophthalmos, blindness of the left eye, drunken gait without sensory-motor deficit and without cervical lymphadenopathy. She had no history of acute or chronic sinusitis. She reported glaucoma of the right eye and a notion of breast neoplasia in the maternal aunt. Seven years earlier (2014), the patient had undergone neo-adjuvant chemotherapy followed by mastectomy with left axillary dissection for a Triple Negative Breast Cancer (TNBC) but without adjuvant radiotherapy. In 2018, she still underwent a first mastectomy with axillary dissection followed by chemotherapy and adjuvant radiotherapy for an Infiltrating duct carcinoma of the contralateral breast. A CT scan of the facial and cerebral mass showed a tissue mass from the left lytic sphenoid sinus invading the ipsilateral temporal lobe with lysis of the pterygoid process with close contact of the palatine tonsil (Figure 1). Histology with immunostaining of parts of the bilateral sphenodotomy concludes with adenocarcinoma of the sphenoid sinus of mammary origin with a triple negative molecular profile (Figure 2). Her extension assessment updated by thoraco-abdominopelvic CT found in addition to the nasosinus location a right thoracic parietal tissue process of $62,5 \times 47 \times 67 \mathrm{~mm}$ with endothoracic development associated with probable secondary pulmonary, ganglion, hepatic and right pleural locations. Palliative hypofractionated radiotherapy of $39 \mathrm{~Gy}$ in 13 fractions is indicated and performed on the secondary sphenoidal location (Figure 3) and systemic treatment with polychemotherapy is initiated. The shortterm evolution is marked by a regression of the left exophthalmos, nasal obstruction and sedation of the hemi-cranias but the patient died 5 months after the diagnosis with a generalized visceral disease despite the implementation of palliative chemotherapy. 


\subsection{Clinical Case 2}

A 60-year-old patient was admitted to the Otorhinolaryngology department and then to radiotherapy at the Dalal Jamm University Hospital in Dakar for a

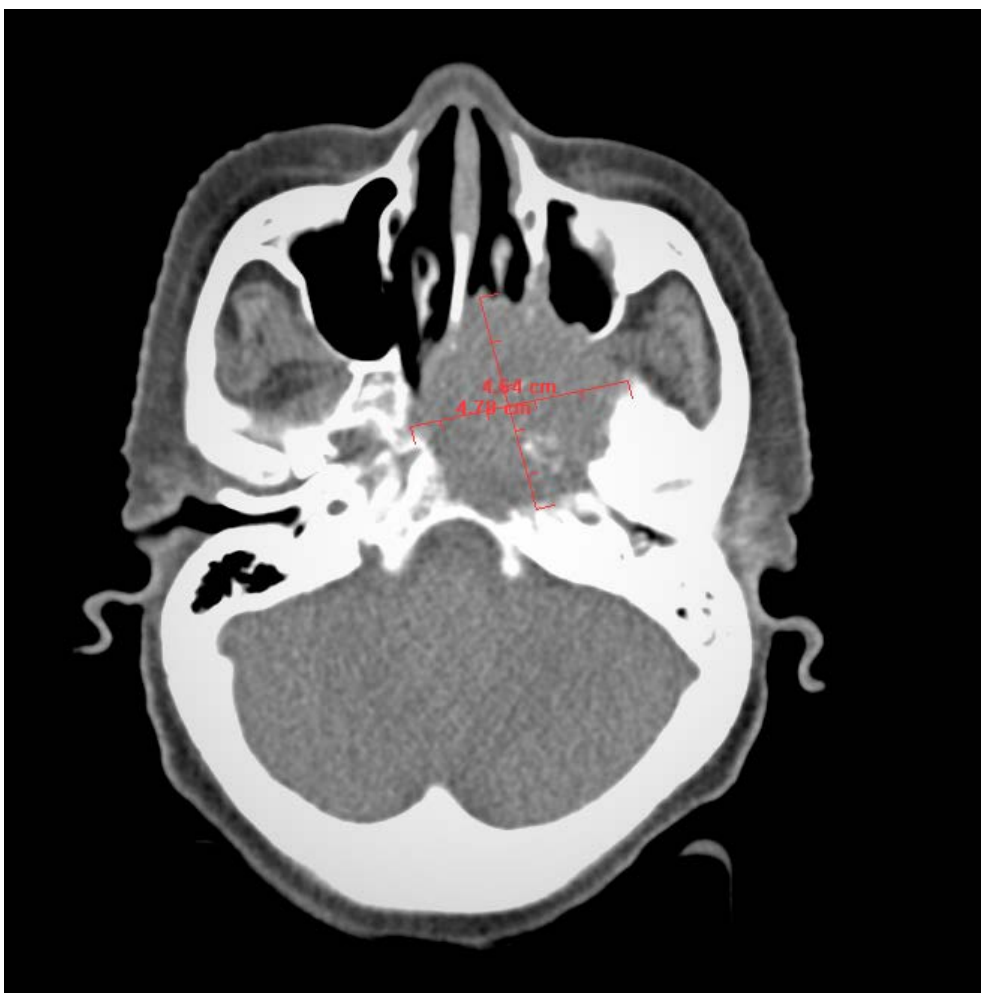

Figure 1. Axial section of a CT scan of the facial mass showing a tissue mass from the left sphenoid sinus with lysis of the ipsilateral pterygoid process.

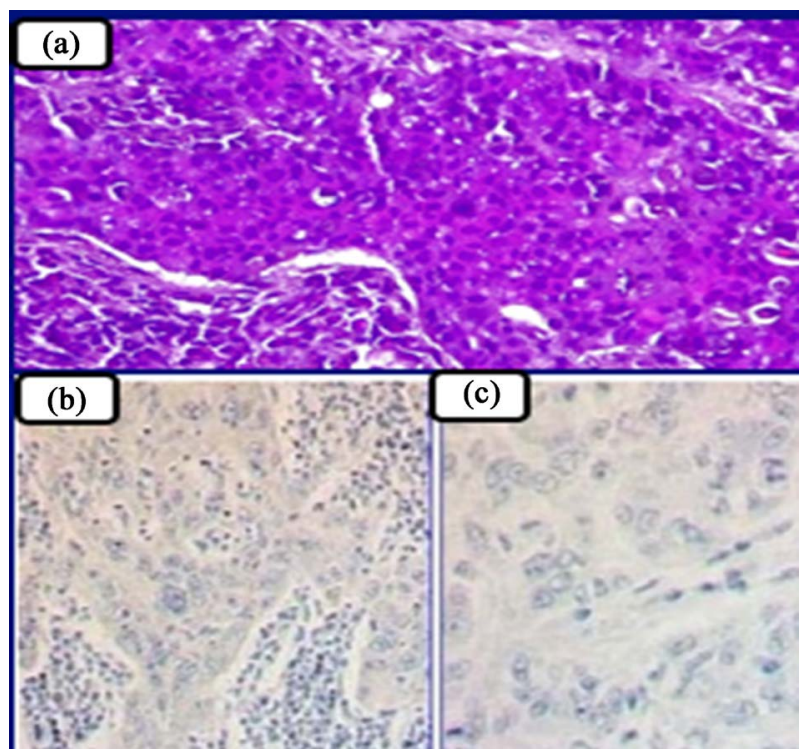

Figure 2. Appearance of sphenoidal metastasis from triple negative breast carcinoma. (a) Histology in favor of invasive carcinoma of breast origin; (b) Immunohistochemical analysis showing hormone receptors (ER, PR) negative (<10\%); (c) Immunohistochemical analysis showing negative HER-2 receptors. 

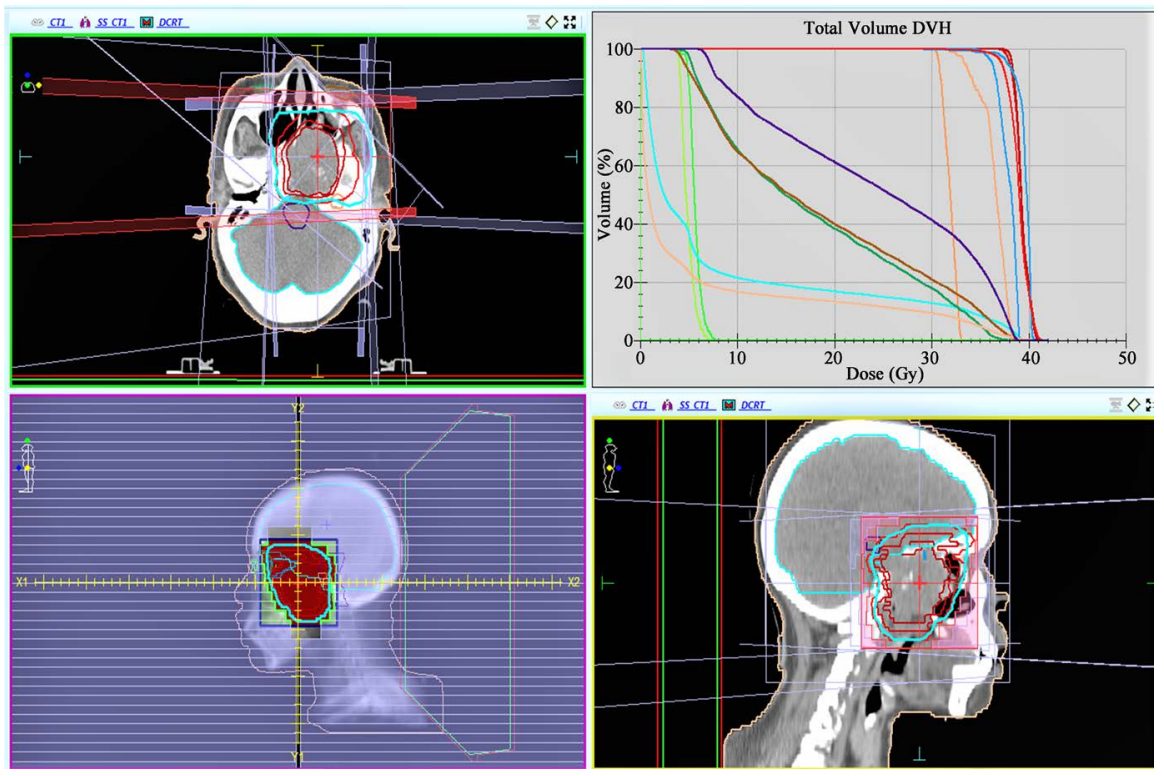

Figure 3. External radiotherapy treatment plan with placement of irradiation and HDV beams.

symptomatology made up of a right unilateral nasal obstruction associated with rhinorrhea and an ipsilateral endonasal mass evolving for 4 months. Two years earlier, the patient had undergone a nodulectomy of the left breast in a non-specialized environment and whose pathological examination confirmed an infiltrating duct carcinoma Scarf-Bloom-Richardson grading II with positive hormone receptors. The patient was then lost to follow-up and did not receive adjuvant treatment. On clinical examination, she presented bilateral paranasal swelling associated with a slight deviation of the nose and a suspicious endonasal mass associated with a movable centimetric submental lymphadenopathy. In the left breast, we noted the presence of an areolo-nipple scar associated with a hard mass at the level of the superior internal quadrant of approximately $2 \mathrm{~cm}$ mobile compared to the two planes associated with a left axillary lymphadenopathy of $2 \mathrm{~cm} \mathrm{mo-}$ bile. A CT scan of the facial mass confirms the lesion invading the nasal cavity, the right maxillary sinus, osteolytic with extension towards the base of the skull and bilateral meningeal (Figure 4). A thoraco-abdominopelvic CT described a malignant infiltrative left retro nipple tumor process associating a carcinomatous nodule of the ipsilateral superior internal quadrant, a left axillary lymphadenopathy and secondary pulmonary and bone locations on the osteolytic thoracic level (T7/T9). The anatomopathological examination of the biopsy specimens from the maxillary sinus is in favor of a secondary localization of a carcinoma of the mammary type expressing the hormonal receptors, with HER negative and Ki $67 \%$ to $20 \%$ (Figure 5). The patient received 30 Gy hypo-fractionated radiotherapy in 10 fractions on the localization of the maxillary sinus and $20 \mathrm{~Gy}$ in 5 fractions on the T7/T9 osteolytic lesion associated with sequential chemotherapy of the type $(4 \mathrm{AC} 60+4$ Taxotere +6 Zometa) followed by left mastectomy of cleanliness. The evolution was marked by a regression of the lesion of the facial 


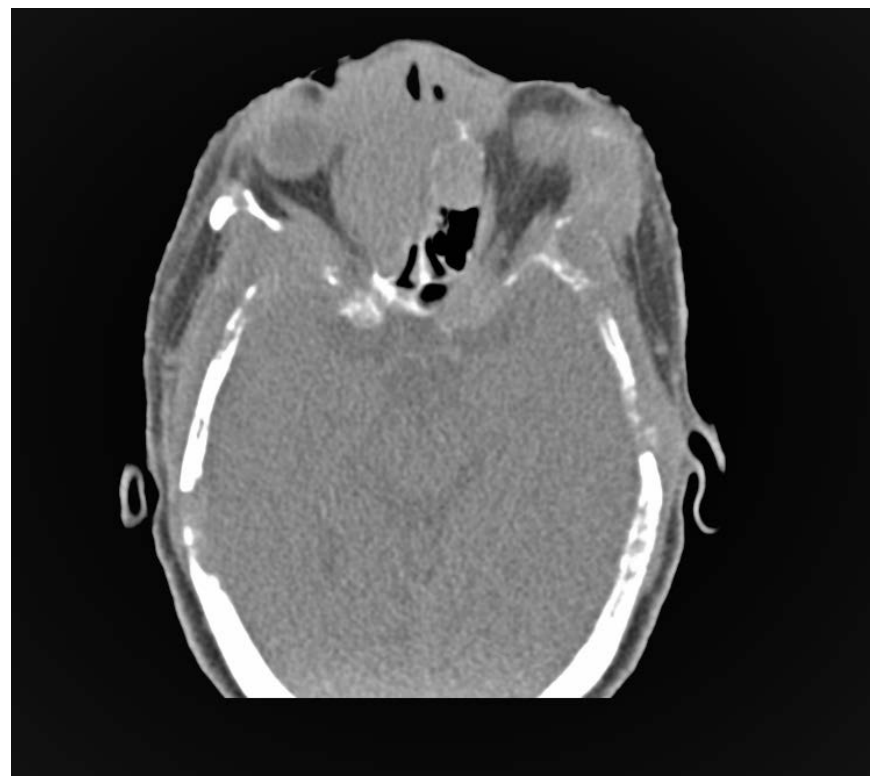

Figure 4. Axial CT scan of the facial mass showing a mass invading the nasal fossa, the right maxillary sinus, osteolytic with extension towards the base of the skull and bilateral meningeal.

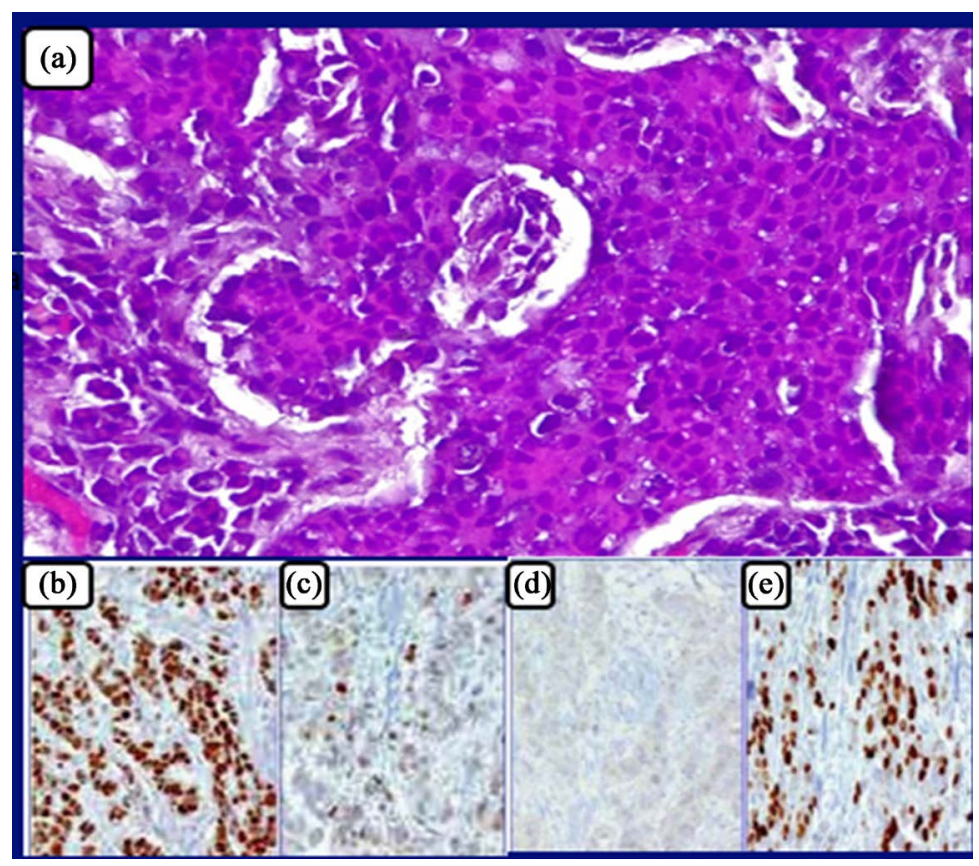

Figure 5. Appearance of metastasis of the maxillary sinus and nasal cavity of mammary carcinoma of luminal B molecular profile. (a) Histology in favor of invasive carcinoma of breast origin; (b) Immunohistochemical analysis showing positive estrogenic hormone receptors (ERs); (c) Immunohistochemical analysis showing positive progesterone hormone receptors (PRs); (d) Immunohistochemical analysis showing negative HER-2 receptors; (e) Immunohistochemical analysis showing a proliferation index greater than $20 \%$.

mass, a stabilization of the other secondary locations, in particular the bone and lungs, and the patient was alive 16 months after the diagnosis of the recurrence 
of her left breast cancer.

\section{Discussion}

Breast cancer in women has now overtaken lung cancer in terms of incidence in 2020 , with around 2.3 million new cases, or $11.7 \%$ of all cancer cases. It is the fifth leading cause of cancer death worldwide, with 685,000 deaths [1]. Improved treatment of this disease led to better survival, indirectly giving more time for the appearance of unusual secondary locations. Hematogenous metastases from breast cancer could affect virtually any anatomical site in the body, but most commonly occur in bone, lungs and liver [4].

Nasosinus metastases from this cancer are rare. It occupies the fourth position as the starting point of secondary tumors of the frontal and ethmoidal sinuses, behind cancers of the kidneys, bronchi, and thyroid gland [7].

The clinical symptoms are not specific and may be confused with those of other tumors in the region of the facial mass. The table consists mainly of mainly unilateral nasal symptoms including rhinorrhea, nasal obstruction and epistaxis, which may be associated with mass syndrome [8], with neurological, ophthalmological or cutaneous manifestations. The proximity to the orbit can then cause compression or invasion leading to ocular symptoms: exophthalmos, diplopia and blindness [7]. In some cases reported in the literature, the symptomatology has wrongly suggested the diagnosis of ethmoiditis [2], treatment-resistant subacute sinusitis, or cavernous sinus thrombophlebitis with neurological and ophthalmological signs [8]. In our patients the clinical picture was revealed by nasal signs such as nasal obstruction, rhinorrhea and a mass syndrome with repercussions on the ophthalmic and neurological level. The radiological aspects are not specific and join those of malignant tumors of the region which classically manifest themselves in the form of a full, homogeneous tumor mass, which is enhanced after injection of iodine, classically osteolytic [9]. It is above all the anamnestic history of the patient, the endoscopic exploration as well as the anatomopathological and immunohistochemical reading of the biopsy samples which confirms the mammary origin of these secondary locations. More generally, the restaging of the patient finds other secondary locations, in particular bone and visceral locations.

The route of dissemination proposed by Batson is the most commonly accepted [5]. According to this theory, the vertebral venous plexus is involved, playing an initial role in the distal metastases of tumor cells. This plexus consists of an epidural venous plexus and a prevertebral venous plexus. These two plexuses are largely interconnected via the intercostal veins and the azygos veins. In this venous plexus devoid of valves, reflux often occurs when intra-abdominal and endothoracic pressures are high. As a result, tumor cells can reach the cavernous sinus, the pterygoid venous plexus and the pharyngeal venous plexus and then migrate further towards the sinuses, the brain and the skull [4]. A route of hematogenous dissemination from occult lung metastases has also been pro- 
posed [8].

The diagnosis of these secondary nasosinus localizations is often concomitant with other metastatic bone and visceral lesions which largely determine the patient's prognosis.

The therapeutic strategy generally includes: local treatment with radiotherapy for analgesia and local control to avoid serious functional or vital complications and systemic treatment with palliative chemotherapy even if the response to systemic treatment remains weak [10]. In our patients, hypofractionated radiotherapy made it possible to obtain a rapid analgesic effect and significant local control even if one of the patients died 5 months after the diagnosis. It is important to emphasize that the prognosis of these patients is poor and that in the majority of published cases the overall survival at 1 year was zero [3] [8] [10]. A patient was still alive in good general condition 16 months after the onset of nasosinus metastases and this after hypo-fractionated local radiotherapy, sequential chemotherapy type 4AC60 +4 Taxotere +6 Zometa and mastectomy.left of cleanliness.

\section{Conclusion}

Nasosinus metastases of breast cancer are very rare but possible, this diagnosis should be evoked before any nasosinus manifestation in a patient with a history of breast cancer, which would make it possible to make an early diagnosis and avoid as much possible as locoregional complications. Despite the poor prognosis generally associated with this picture due to generalized disease, radiotherapy offers good local control.

\section{Ethical Considerations}

The informed consent of the two patients was obtained at the diagnosis before the writing of these clinical cases.

\section{Conflicts of Interest}

The authors declare no conflicts of interest regarding the publication of this paper.

\section{References}

[1] Sung, H., Ferlay, J., Siegel, R.L., Laversanne, M., Soerjomataram, I., Jemal, A., et al. (2021) Global Cancer Statistics 2020: GLOBOCAN Estimates of Incidence and Mortality Worldwide for 36 Cancers in 185 Countries. CA: A Cancer Journal for Clinicians, 71, 209-249. https://doi.org/10.3322/caac.21660

[2] Pitkaranta, A., Markkola, A. and Malmberg, H. (2001) Breast Cancer Metastasis Presenting as Ethmoiditis. Rhinology, 39, 107-108.

[3] Austin, J.R., Kershiznek, M.M., McGill, D. and Austin, S.G. (1995) Breast Carcinoma Metastatic to Paranasal Sinuses. Head \& Neck, 17, 161-165.

https://doi.org/10.1002/hed.2880170217

[4] Darouassi, Y., Fetohi, M., Touiheme, N., Ichou, M. and Abrouq, A. (2010) Nasosinus 
Metastasis of Breast Cancer in a Man. La Presse Médicale, 39, 1340-1342.

https://doi.org/10.1016/j.lpm.2010.06.001

[5] Batson, O.V. (1940) The Function of the Vertebral Veins and Their Role in the Spread of Metastases. Annals of Surgery, 112, 138-149.

https://doi.org/10.1097/00000658-194007000-00016

[6] Nelson, E.G., Goldman, M.E. and Hemmati, M. (1990) Metastatic Carcinoma of the Ethmoid Sinus. Otolaryngology_Head and Neck Surgery, 103, 120-123. https://doi.org/10.1177/019459989010300119

[7] Asproudis, I., Gorezis, S., Charalabopoulos, K., Stefaniotou, M., Peschos, D., Psilas, K. (2004) Breast Carcinoma Metastasis to the Orbit and Paranasal Sinuses: A Case Report. Experimental Oncology, 26, 246-248.

[8] Monserez, D., Vlaminck, S., Kuhweide, R. and Casselman, J. (2001) Symmetrical Ethmoidal Metastases from Ductal Carcinoma of the Breast, Suggesting Transcribrosal Spread. Acta Otorhinolaryngologica Belgica, 55, 251-257.

[9] Pignataro, L., Peri, A. and Ottaviani, F. (2001) Breast Carcinoma Metastatic to the Ethmoid Sinus: A Case Report. Tumori Journal, 87, 455-457. https://doi.org/10.1177/030089160108700621

[10] Szmeja, Z., Citowicki, W., Muszyńska, B. and Mielcarek-Kuchta, D. (1998) Breast Carcinoma Metastasis to Paranasal Sinuses. Otolaryngologia Polska, 52, 467-470. 